\title{
GENERATORS FOR $G$ BORDISM
}

\section{R. PAUL BEEM}

\begin{abstract}
We show that a certain collection of $G$ manifolds generates $N_{*}(G)$ as an algebra over $N_{*}$, the unoriented bordism ring, where $G$ is finite abelian and of order not divisible by four.
\end{abstract}

1. Introduction. The object of this paper is to show that a certain natural collection of $G$ manifolds multiplicatively generates the unoriented $G$ bordism ring $N_{*}(G)$ as an algebra over the unoriented bordism ring $N_{*}$, for those finite abelian groups $G$ such that $[G: 1]$ is not a multiple of four. In $\$ \S 2$ and 3 we will give a precise statement of the theorem for odd order groups and even order groups, respectively. For the remainder of the paper, all groups will be finite abelian and of order not divisible by four.

In the rest of this section, we will define terms, establish notation and recall results which we will need.

$\mathscr{F}$ (or $\mathscr{F}^{\prime}, \mathscr{F}_{1}$, etc.) will denote some collection of subgroups of $G$, possibly empty. To be a "family in $G$ ", $\mathscr{F}$ must have the property that $H \in \mathcal{F}$ and $K \subseteq H$, for $K$ a subgroup of $G$, must imply that $K \in \mathcal{F}$. (For this definition and as a general reference, see [4].) $N_{*}\left(G ; \mathcal{F}, \mathcal{F}^{\prime}\right)$ will denote the bordism algebra of smooth $G$ actions on unoriented smooth manifolds, such actions having the property that the isotropy subgroups of points of $M$ (resp. $\partial M$ ) lie in $\mathcal{F}$ (resp. $\mathcal{F}^{\prime}$ ). If "All" denotes the family of all subgroups of $G$, then we denote $N_{*}(G ;$ All, $\phi)$ by $N_{*}(G) . N_{*}\left(G ; \mathscr{F}, \mathscr{F}^{\prime}\right)(X)$, where $X$ is a $G$ space, is the bordism module of $G$ maps of $G$ manifolds (with the $\left(\mathscr{F}, \mathscr{F}^{\prime}\right)$ isotropy condition) into $X$.

We recall certain other notions of [4] which we need here. Two families $\mathscr{F} \supseteq \mathscr{F}^{\prime}$ in $G$ are called "adjacent" if they differ by a single group. There is the $G$-space $B O_{k}(G)$ and a $k$-plane bundle $E_{k}(G)$ with $G$ action and projection $p_{k}: E_{k}(G) \rightarrow B O_{k}(G)$ which is a $G$ map. These are formed as follows. Let $\left\{V_{1}, \ldots, V_{s}\right\}$ be the collection of all irreducible real $G$ representations. Let

$$
V(G)=\left(\bigoplus_{i=1}^{\infty}\left(V_{1}\right)_{i}\right) \oplus \cdots \oplus\left(\bigoplus_{i=1}^{\infty}\left(V_{s}\right)_{i}\right),
$$

where each $\left(V_{j}\right)_{i}$ is a copy of $V_{j}$. This has a natural orthogonal action of $G$. Hence the $k$ planes through the origin in $V(G)$ also receive a $G$ action.

Received by the editors May 16, 1977.

AMS (MOS) subject classifications (1970). Primary 57D85.

Key words and phrases. Equivariant bordism. 
$B O_{k}(G)$ is this space. $E_{k}(G) \subseteq B O_{k}(G) \times V(G)$ and consists of those pairs $(\alpha, \nu)$ with $\nu \in \alpha$. The diagonal action on $B O_{k}(G) \times V(G)$ restricts to $E_{k}(G)$ and the projection on the first factor gives $p_{k}$. This bundle is universal for $k$-plane bundles with $G$ action over reasonable $G$ spaces (certainly for manifolds). We will let $F_{H}^{\prime}\left(B O_{k}(G)\right)$ denote the points of $B O_{k}(G)$ which are left fixed by $H$ (for $H$ a subgroup of $G$ ) and over which $E_{k}(G)$ has no trivial $H$ representation summand.

Then, according to $[4$, p. 20$]$, for adjacent families $\mathscr{F} \supseteq \mathcal{F}^{\prime}$, differing by the group $H$, there is an isomorphism

$$
f_{H}: N_{*}\left(G ; \mathcal{F}, \mathscr{F}^{\prime}\right) \rightarrow \bigoplus_{k=0}^{*} N_{*-k}(G / H ;\{\{1\}\}, \phi)\left(F_{H}^{\prime}\left(B O_{k}(G)\right)\right) .
$$

The homomorphism assigns to a $G$ action the classifying map of the normal bundle to the fixed set of the included $H$ action. (Note that the above fixed point homomorphism sends the cartesian product algebra structure of the left-hand side to the multiplication induced by Whitney sums of vector bundles on the right-hand side.)

Finally, we say that a pair $\left(\mathscr{F}, \mathscr{F}^{\prime}\right)$ are "quasi-adjacent" with respect to a collection $\mathbb{Q} \subseteq \mathscr{F}$ if

(i) $H \neq H^{\prime}$ and $H, H^{\prime}$ in $\mathbb{Q}$ imply that $\operatorname{grp}\left(H \cup H^{\prime}\right) \notin \mathscr{F}$;

(ii) $L \in \mathscr{F}-\mathscr{F}^{\prime}$ implies $\exists H \in \mathbb{Q}$ with $H \subseteq L$.

In this case, there is an isomorphism

$$
N_{*}\left(G ; \mathscr{F}, \mathscr{F}^{\prime}\right) \rightarrow \bigoplus_{H \in \mathfrak{Q}} \bigoplus_{k=0}^{*} N_{*-k}\left(G / H, \mathscr{F}_{H}, \mathscr{F}_{H}^{\prime}\right)\left(F_{H} B O_{k}(G)\right),
$$

where $\left(\mathscr{F}_{H}, \mathscr{F}_{H}^{\prime}\right)$ contain subgroups whose preimages are in $\left(\mathscr{F}, \mathscr{F}^{\prime}\right)$. See [4] as usual.

The author wishes to thank R. E. Stong for mentioning this problem and for his helpful suggestions.

2. Groups of odd order. Let $x(K)$ denote the bordism class of the factor group $G / K$ with $G$ action. $x(K) \in N_{0}(G)$ for every subgroup $K$ in $G$. Let $\left\{\theta_{i}\right.$ : $i=1,2, \ldots, r\}$ denote a complete set of nontrivial, irreducible complex representations of $G$, thought of as homomorphisms from $G$ into $S^{1}=U(1)$, the circle group. Let $E_{n}$ denote the total space of the Hopf line bundle over $C P(n)$. Then $G$ acts on $E_{n}$ via $\theta_{i}$. Let $Q(2 n+2, i)$ denote the $G$ manifold, $R P\left(E_{n} \oplus 1\right)$, with $G$ action induced by $\left(\theta_{i} \oplus\right.$ trivial $)$.

THEOREM 1. $N_{*}(G)$ is multiplicatively generated by $\{x(K): K$ is a subgroup of $G\}$ and $\{Q(2 n+2, i): n=0,1, \ldots ; i=1,2, \ldots, r\}$, as an algebra over $N_{*}$.

Proof. First we construct an ascending chain of pairwise adjacent families in $G$ from the empty family to All.

Let $\mathscr{P}_{0}=$ All $-\{G\}$. Having $\mathscr{P}_{i}$, let $\mathscr{P}_{i+1}=\mathscr{P}_{i}-\{$ maximal subgroups in $\left.\mathscr{P}_{i}\right\}$. Suppose $\mathscr{P}_{n}=\varnothing$. Now, for each $i$ adjoin one at a time to $\mathscr{P}_{i-1}$, the 
elements of $\mathscr{P}_{i}-\mathscr{P}_{i-1}$ (in any order) to get

$$
\mathscr{P}_{i-1}=Q_{i, 1} \subsetneq \cdots \underset{\ddagger}{\subsetneq} Q_{i, n_{i}}=\mathscr{P}_{i} .
$$

The collection $\left\{Q_{i, j}\right\}$ works.

If $\mathscr{F} \supseteq \mathcal{F}^{\prime}$ are any successive families in this collection, which differ by $K$, then

$$
N_{*}\left(G ; \mathscr{F}, \mathscr{F}^{\prime}\right)=\bigoplus_{k=0}^{*} N_{*-k}(G / K ; \text { Free })\left(F_{K}^{\prime}\left(B O_{k}(G)\right)\right)
$$

where "Free" $=\{\{1\}\}$. This equals $[4$, p. 20]

$$
\bigoplus_{k=0}^{*} N_{*-k}\left(E(G / K) \underset{(G / K)}{\times} F_{K}^{\prime}\left(B O_{k}(G)\right)\right)
$$

where $E(G / K)$ denotes the total space of the universal principal $G / K$ bundle. But, since $G / K$ is an odd order group (also see $\S 3$ ),

$$
E(G / K) \underset{(G / K)}{\times} F_{K}^{\prime}\left(B O_{k}(G)\right) \approx B(G / K) \times F_{K}^{\prime}\left(B O_{k}(G)\right) ;
$$

see [2, Proposition 3.2]. Hence, the above sum is

$$
\bigoplus_{k=0}^{*} N_{*-k}\left(B(G / K) \times F_{K}^{\prime}\left(B O_{k}(G)\right)\right),
$$

which is

$$
N_{*}(B(G / K)) \otimes_{N_{*}}\left(\bigoplus_{k=0}^{*} N_{*-k}\left(F_{K}^{\prime}\left(B O_{k}(G)\right)\right)\right)
$$

or just

$$
\bigoplus_{k=0}^{*} N_{*-k}\left(f_{K}^{\prime}\left(B O_{k}(G)\right)\right)
$$

since $N_{*}(B(G / K)) \cong N_{*}$, the unoriented bordism ring $(G / K$ having odd order).

Since $K$ has odd order, the nontrivial irreducible representations are all complex and $E_{k}(G)$ splits over $F_{K}^{\prime}\left(B O_{k}(G)\right)$ into a sum of complex bundles. Since $F_{K}^{\prime}\left(B O_{k}(G)\right)$ is a universal space, it must be a disjoint union of products $B U_{n_{1}} \times \cdots \times B U_{n_{q}}$, where $q$ is the number of distinct nontrivial irreducible representations of $K$ and $n_{1}+\cdots+n_{q}=k / 2$. $K$ acts in the fibres over $B U_{n_{1}}$ via its $i$ th irreducible representation.

Putting all this together, then, there is an isomorphism of algebras

$$
\rho: N_{*}\left(G ; \mathcal{F}, \mathcal{F}^{\prime}\right) \rightarrow \bigoplus_{k=0}^{[* / 2]} \bigoplus_{(n)} N_{*-2 k}\left(B U_{n_{1}} \times \cdots \times B U_{n_{q}}\right) \text {, }
$$

where $(n)$ denotes the sequence $\left(n_{1}, \ldots, n_{r}\right)$ and the sum is over all $(n)$ with $n_{1}+\cdots+n_{q}=k / 2$.

We want to consider all exact sequences of the form

$$
\cdots \underset{\partial}{\rightarrow} N_{*}\left(G ; \mathscr{F}^{\prime}, \phi\right) \underset{i}{\rightarrow} N_{*}(G ; \mathscr{F}, \phi) \underset{j}{\rightarrow} N_{*}\left(G ; \mathscr{F}, \mathscr{F}^{\prime}\right) \underset{\partial}{\rightarrow} \ldots,
$$


where $i$ and $j$ are induced by inclusions of families and $\partial(M, \psi)=$ $(\partial M, \psi / \partial M)$. (See [4, p. 6], for a proof of exactness.) We assume that $\mathscr{F} \supseteq \mathscr{F}^{\prime}$ are an adjacent pair of families from our previously constructed collection and that $N_{*}\left(G ; \mathscr{F}^{\prime}, \phi\right)$ is generated over $N_{*}$ by the products $[G / H$, $G][Q(m, i)]$, where $H \in \mathscr{F}^{\prime}$. (Since $N_{*}(G ;$ Free $) \cong N_{*}$, our induction gets started.)

Let $K \in \mathscr{F}-\mathscr{F}^{\prime}$. Note that

$$
\rho(j[(G / K) \times Q(2 n+2, i)])=\left[C P(n) \subset B U_{1}\right] .
$$

As $i$ varies over all the homomorphisms from $G$ to $S^{1}$, the actions of $K$ on $E_{n}$ will vary over all its irreducible representations. Hence, the inclusions $C P(n)$ $\subset B U_{1}$ will vary over all the inclusions. But these maps generate (in fact, they are an algebra basis) the image of $\rho$.

It follows that $N_{*}(G ; \mathscr{F}, \phi)$ is algebraically generated by the classes $[(G / K) \times Q(2 n+2, i)]$, for $K \in \mathscr{F}$. This completes the proof.

3. $[G: 1] \equiv 2(\bmod 4)$. Write $G=H \times Z_{2}$, where $H$ has odd order. Let $\left\{\theta_{i}: i=1,2, \ldots, r\right\}$ denote a complete set of nontrivial irreducible complex representations of $H$ as in $\S 2$. Let $P(n, k, i)$ denote $R P\left(E_{n} \oplus k \oplus 1\right)$, where the $H$ action is induced by $\left(\theta_{i} \oplus\right.$ trivial $\oplus$ trivial), and the $Z_{2}$ action is generated by $(-1) \oplus(-1) \oplus(1)$. Let $Q(2 n+2, i)$ denote $R P\left(E_{n} \oplus 1\right)$ with $H$ given by $\theta_{i} \oplus 1$ and trivial $Z_{2}$ action.

THEOREM 2. $N_{*}(G)$ is multiplicatively generated by $\{x(K): K$ is a subgroup of $G$ and $[G: K]$ is odd $\},\{Q(2 n+2, i): n=0,1, \ldots ; i=1,2, \ldots, r\}$, $\{P(n, k, i): n=0,1, \ldots ; i=1,2, \ldots, r ; k=0,1,2, \ldots\}$ and the image of $N_{*}\left(Z_{2}\right)$ in $N_{*}(G)$ obtained by letting $H$ act trivially on $Z_{2}$ manifolds.

Proof. Let $\mathscr{F}$ denote $\left\{K \subseteq G: K \cap Z_{2}=\{0\}\right\}$, which is a family of subgroups of $G$. There is an exact sequence

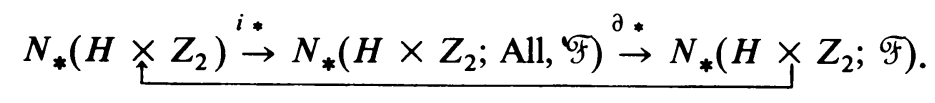

Let $(M, \phi)$ denote an $\mathscr{F}$-free $H \times Z_{2}$ action and give the interval $I=[-1,1]$ an $H \times Z_{2}$ action by letting the generator of $Z_{2}$ act by multiplication by -1 and the elements of $H$ act trivially. Then the twisted product $M \times_{\left(H \times Z_{2}\right)} I$ has a natural (All, $\mathscr{\mathscr { F }}$ )-free $H \times Z_{2}$ action and boundary $(M, \phi)$. Since all is well defined on bordism, $\partial_{*}$ is an epimorphism.

Lemma 2.1. $N_{*}\left(H \times Z_{2} ; \mathscr{F}\right) \cong N_{*}(H) \otimes_{N_{*}} N_{*}\left(Z_{2}\right.$; Free) as $N_{*}$ algebras.

Proof. Let $\Delta$ denote the Smith homomorphism associated with the nontrivial 1-dimensional representation of $G$ [3]; then there is an exact sequence:

$$
\stackrel{N_{*}(H) \stackrel{\text { extension }}{\rightarrow}}{\rightarrow} N_{*}\left(H \times Z_{2} ; \mathscr{F}\right) \stackrel{\Delta}{\rightarrow} N_{*}\left(H \times Z_{2} ; \mathscr{F}\right) .
$$

Since restriction is clearly zero, $\Delta$ is an epimorphism. 
There is also the usual short exact sequence, where $S$ denotes the usual Smith homomorphism:

$$
0 \rightarrow N_{*} \rightarrow N_{*}\left(Z_{2} ; \text { Free }\right) \stackrel{s}{\rightarrow} N_{*}\left(Z_{2} ; \text { Free }\right) \rightarrow 0 .
$$

Tensoring this latter sequence with $N_{*}(H)$, a free $N_{*}$ module and mapping it by the product homomorphism into the first sequence, we get:

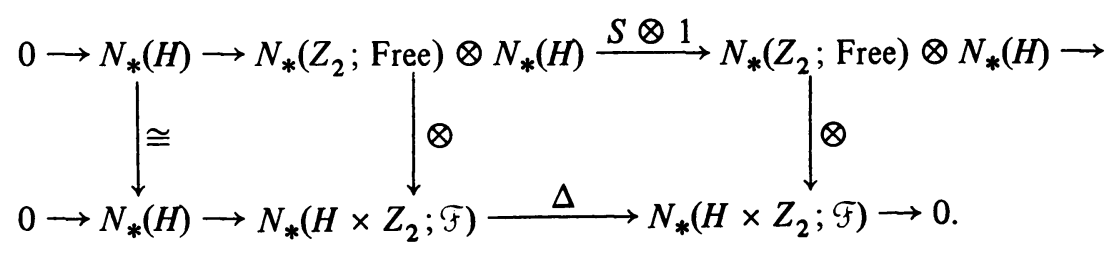

An easy induction, using the Five-Lemma, completes the proof.

There are homomorphisms

$$
N_{*}(H)\left(B O_{1}, \text { trivial }\right) \underset{f}{\stackrel{g}{\rightleftarrows}} N_{*}\left(H \times Z_{2} ; \mathscr{F}\right),
$$

which, it is easy to show using Lemma 2.1 , are inverse isomorphisms. $g$ is given by a sphere bundle construction and $f$ classifies the free involution.

Since All and $\mathscr{F}$ are quasi-adjacent families in $H \times Z_{2}$ with respect to $\left\{Z_{2}\right\}$

$$
N_{*}\left(H \times Z_{2} ; \text { All, } \mathscr{F}\right)=\bigoplus_{n=0}^{*} N_{*-n}(H)\left(B O_{n}(H)\right)
$$

as algebras and $\partial_{*}$ becomes

$$
\partial_{*}^{\prime}: \bigoplus_{n=0} N_{*-n}(H)\left(B O_{n}(H)\right) \rightarrow N_{*}(H)\left(B O_{1} \text {, trivial }\right)
$$

given by the usual projective bundle construction.

Next we look at the (All, Free) exact sequence for the $H$ bordism of the spaces $B O_{n}(H)$. We get

$$
\begin{aligned}
& \cdots \rightarrow \bigoplus N_{*-n}(H ; \text { Free })\left(B O_{n}(H)\right) \stackrel{\alpha}{\rightarrow} \bigoplus N_{*-n}(H)\left(B O_{n}(H)\right) \\
& \stackrel{\beta}{\rightarrow} \bigoplus N_{*-n}(H ; \text { All; Free })\left(B O_{n}(H)\right) \rightarrow \ldots,
\end{aligned}
$$

an exact sequence of algebras.

LEMMA 2.2. $\alpha$ is a monomorphism and the image of $\alpha$ is precisely the image of extension: $N_{*}\left(Z_{2} ;\right.$ All, Free $) \rightarrow N_{*}\left(H \times Z_{2} ;\right.$ All, F $)$.

Proof. $N_{*}(H$; Free $)\left(B O_{n}(H)\right) \cong N_{*}\left(E H \times_{H} B O_{n}(H)\right)$. Clearly $E H$ $\times_{H} E_{n}(H)$ is an $n$-plane bundle over $E H \times_{H} B O_{n}(H)$. Classifying this and projecting to $B H$ gives a homotopy equivalence

$$
E H \times{ }_{H} B O_{n}(H) \rightarrow B H \times B O_{n} .
$$


Since $H$ has odd order, $N_{*}\left(B H \times B O_{n}\right) \cong N_{*}\left(B O_{n}\right)$.

The composite isomorphism

$$
j_{*}: \bigoplus N_{*-n}(H ; \text { Free })\left(B O_{n}(H)\right) \rightarrow \bigoplus N_{*-n}\left(B O_{n}\right)
$$

takes the $n$-plane bundle $\eta$ over $M$ to $\eta / H$ over $M / H$.

There is also the extension homomorphism $e$ which sends an $n$-plane bundle $\eta$ over $M$ to $\eta \times H$ over $M \times H$. Since $j_{*} e$ is the identity, $e$ is an isomorphism.

There is also a forgetful homomorphism

$$
\rho: \bigoplus N_{*-n}(H)\left(B O_{n}(H)\right) \rightarrow \bigoplus N_{*-n}\left(B O_{n}\right)
$$

with $\rho \alpha=j$. Hence, $\alpha$ is a monomorphism and image $\alpha$ is $Z_{2}$ extensions. This proves 2.2.

Therefore, $N_{*}\left(H \times Z_{2}\right.$; All, $\left.\mathscr{F}\right)$, modulo the ideal of $Z_{2}$ extensions (from $N_{*}\left(Z_{2}\right.$; All, Free)), is isomorphic as $N_{*}$ algebras to $\bigoplus_{n=0}^{*} N_{*-n}(H$; All, Free) $\left(B O_{n}(H)\right)$. In $N_{*}\left(H \times Z_{2}\right)$, the ideal of $Z_{2}$ extensions is given by products of $Z_{2}$ classes with $x\left(Z_{2}\right)$. Let

$$
\mathscr{F}_{r}=\text { All } \supsetneqq \cdots \underset{\not}{\supsetneq} \mathscr{F}_{i+1} \supsetneqq \mathscr{F}_{i} \supsetneq \cdots \cdots \underset{\not}{\supsetneq} \mathscr{F}_{1}=\text { Free }
$$

be a sequence of successively adjacent families in $H$. We wish to consider all sequences

$$
\begin{aligned}
\cdots \rightarrow N_{*}\left(H ; \mathscr{F}_{i}, \mathscr{F}_{1}\right)\left(B O_{n}(H)\right) \stackrel{\gamma}{\rightarrow} N_{*}\left(H ; \mathscr{F}_{i+1}, \mathscr{F}_{1}\right)\left(B O_{n}(H)\right) \\
\qquad \stackrel{\delta}{\rightarrow} N_{*}\left(H ; \mathscr{F}_{i+1}, \mathscr{F}_{i}\right)\left(B O_{n}(H)\right) \rightarrow \cdots
\end{aligned}
$$

and wish to establish the claim that $N_{*}\left(H ; \mathscr{F}_{i+1}, \mathscr{F}_{1}\right)\left(B O_{n}(H)\right)$ is generated by our potential generators (together with classes which split back from $N_{*}\left(H \times Z_{2}, \mathscr{F}\right)$ ) if $N_{*}\left(H ; \mathscr{F}_{i}, \mathscr{F}_{1}\right)$ is. (Since $\mathscr{F}_{2}$ differs from $\mathscr{F}_{1}$ by a single group, the proof of the induction step will also get the induction started.)

LEMMA 2.3.

$$
\begin{aligned}
& \bigoplus_{n=0}^{*} N_{*-n}\left(H ; \mathscr{F}_{i+1}, \mathscr{F}_{i}\right)\left(B O_{n}(H)\right) \cong\left(\bigoplus_{r=0}^{*} N_{*-r}\left(F_{K}^{\prime} B O_{r}(H)\right)\right) \\
& \otimes\left(\bigoplus_{s=0}^{*} N_{*-s}\left(F_{K}^{\prime} B O_{s}(H)\right)\right) \otimes\left(\bigoplus_{t=0}^{*} N_{*-t}\left(B O_{t}\right)\right),
\end{aligned}
$$

where $\{K\}=\mathscr{F}_{i+1}-\mathscr{F}_{i}$.

Proof. According to [4], $N_{*-n}\left(H ; \mathscr{F}_{i+1}, \mathscr{F}_{i}\right)\left(B O_{n}(H)\right)$ is isomorphic to

which is 


$$
\bigoplus_{k=0}^{*-n} N_{*-n-k}\left(\left(F_{K} B O_{n}(H) \times F_{K}^{\prime} B O_{k}(H)\right) \times_{H / K} E(H / K)\right) .
$$

It is possible to change the $H$ action in the universal bundle over $F_{K} B O_{n}(H)$ $\times F_{K}^{\prime} B O_{k}(H)$ to make $K$ act trivially and for the resulting $H / K$ action to cover the base. Hence,

$$
\begin{aligned}
\bigoplus_{n=0}^{*} N_{*-n}\left(H ; \mathscr{F}_{i+1}, \mathscr{F}_{i}\right)\left(B O_{n}(H)\right) \\
\quad \bigoplus_{n=0}^{*} \bigoplus_{k=0}^{*-n} N_{*-n-k}\left(F_{K} B O_{n}(H) \times F_{K}^{\prime} B O_{k}(H)\right) .
\end{aligned}
$$

It follows from $\S 1$ that $F_{K} B O_{n}(H)$ is a disjoint union of all products $B O_{t} \times B U_{n_{1}} \times \cdots \times B U_{n_{p}}$, where $t+2 n_{1}+\cdots+2 n_{p}=n$ and $p$ is the number of distinct nontrivial irreducible complex representations of $K$. Let $(n)=\left(n_{1}, n_{2}, \ldots, n_{p}\right)$ and $|n|=n_{1}+\cdots+n_{p}$; then

$$
F_{K} B O_{n}(H)=\bigcup_{s+2|n|=n} B O_{s} \times B U_{(n)}
$$

and

$$
F_{K}^{\prime} B O_{k}(H)=\bigcup_{2|q|=k} B U_{(q)}
$$

The Künneth Theorem finishes the proof of 2.3.

Denote $Q(2 n+2, i)$ by $x(n, i)$ and $P(n, k, i)$ by $y(n, k, i)$. There are the following classes in

$$
\begin{gathered}
A_{*}(K)=\left(\bigoplus_{j=0}^{*} N_{*-j}\left(F_{K}^{\prime} B O_{j}(H)\right)\right) \otimes\left(\bigoplus_{k=0}^{*} N_{*-k}\left(F_{K}^{\prime} B O_{k}(H)\right)\right) \\
\otimes\left(\bigoplus_{l=0}^{*} k N_{*-l}\left(B O_{l}\right)\right) .
\end{gathered}
$$

The first factor is generated by the bundle-bordism classes $\alpha(n, j)=$ class of the Hopf bundle over $\mathrm{CP}(n)$ with $H$ acting via $\theta_{j}$. The second factor is generated by $\beta(n, j)$ defined exactly as $\alpha(n, j)$, and the third factor is generated by $\gamma(n)=$ class of the twisted line bundle over $\mathbf{R} P(n-1)$. Finally, we need the following convenient generating set for $N_{*}\left(Z_{2}\right)$ [1]. Let $z(j)$ be the class of the manifold $\mathbf{R} P(j)$ with involution, $\left[r_{0} ; r_{1}: \cdots: r_{j}\right] \rightarrow\left[-r_{0}\right.$ : $\left.r_{1}: \cdots: r_{j}\right]$ and $K_{1}$ the $N_{*}$ endomorphism on $N_{*}\left(Z_{2}\right)$ given on $Z_{2}$ manifolds by $K_{1}(M, T)=\left(\left(M \times S^{1}\right) /(T \times(-1)), 1 \times\right.$ conjugation $)$. It is known that the classes $\left(K_{1}^{k} z\left(j_{1}\right)\right) z\left(j_{2}\right) \cdots z\left(j_{n}\right)=z\left(j_{1}, \ldots, j_{n}, k\right)$ generate $N_{*}\left(Z_{2}\right)$.

It is clear that $A_{*}(K)$ is generated as a $N_{*}$ module by monomials of the form $\gamma(1)^{k} \gamma\left(h_{1}\right) \cdots \gamma\left(h_{n}\right) \alpha\left(n_{1}, j_{1}\right) \cdots \alpha\left(n_{m}, j_{m}\right) \beta\left(m_{1}, l_{1}\right) \cdots \beta\left(m_{p}, l_{p}\right)$, where $k \geqslant 0 ; 1<h_{1} \leqslant h_{2} \leqslant \cdots \leqslant h_{n}$. (We include the possibility that $n$ (resp. $m$ or $p$ ) may be zero, in which case no $\gamma\left(h_{j}\right)$ (resp. $\alpha$ or $\beta$ ) may appear.)

Consider the following collection of cases: 
1. $n \neq 0$

2. $n=0$ and $k=0$;

3. $n=0, k \neq 0, p=0$;

4. $n=0, k \neq 0, p \neq 0$.

Case 1. Consider the class

$$
\beta i_{*}\left(x(K) z\left(h_{1}, \ldots, h_{n}, k\right)\left(\prod_{i=1}^{m} x\left(n_{i}, j_{i}\right)\right)\left(\prod_{i=1}^{p} y\left(m_{i}, 0, l_{i}\right)\right)\right] .
$$

Its image in $A_{*}(K)$ is [1]

$$
\begin{aligned}
\left(\gamma(1)^{k} \gamma\left(h_{1}\right) \cdots \gamma\left(h_{n}\right)+\Gamma\right)\left(\alpha\left(n_{1}, j_{1}\right)\right. & \left.\cdots \alpha\left(n_{m}, j_{m}\right)\right) \\
& \times\left(\beta\left(m_{1}, l_{1}\right) \cdots \beta\left(m_{p}, l_{p}\right)\right),
\end{aligned}
$$

where $\Gamma$ is a sum of monomials of the form $\gamma(1)^{r} \gamma\left(s_{1}\right) \cdots \gamma\left(s_{t}\right)$ where $r>k$. These images therefore generate this case.

Case 2.

$$
\beta i_{*}\left(x(K)\left(\prod_{i=1}^{m} x\left(n_{i}, j_{i}\right)\right)\left(\prod_{i=1}^{p} y\left(m_{i}, 0, l_{i}\right)\right)\right\}
$$

generate this case.

Case 3. By Lemma 2.1, these classes split back from $N_{*}\left(H \times Z_{2} ; \mathscr{F}\right)$.

Case 4. We want to show that we can hit classes of the form:

$$
\gamma(1)^{k} \alpha\left(n_{1}, j_{1}\right) \cdots \alpha\left(n_{m}, j_{m}\right) \beta\left(m_{1}, l_{1}\right) \cdots \beta\left(m_{p}, l_{p}\right)
$$

where $p>1$. We induct on $p$. If $p=1$, we consider the class

$$
\beta i_{*}\left(x(K)\left(\prod_{i=1}^{m} x\left(n_{i}, j_{i}\right)\right) y\left(m_{1}, k, l_{1}\right)\right)
$$

in $A_{*}(K)$, obtaining $\left(\gamma(1)^{k} \beta\left(m_{1}, l_{1}\right)+\Gamma\right) \alpha\left(n_{1}, j_{1}\right) \cdots \alpha\left(n_{m}, j_{m}\right)$, where $\Gamma$ is a line bundle over $\mathbf{R} P\left(E_{m_{1}} \oplus k\right)$. Hence we can hit this class with the help of classes from $N_{*}\left(H \times Z_{2} ; \mathcal{F}\right)$. For $p>1$,

$$
i_{*}\left(x(K)\left(\prod_{i=1}^{m} x\left(n_{i}, j_{i}\right)\right) y\left(m_{1}, k, l_{1}\right)\left(\prod_{i=2}^{p} y\left(m_{i}, 0, l_{i}\right)\right)\right]
$$

is, in $A_{*}(K)$,

$$
\prod_{i=1}^{m} \alpha\left(n_{i}, j_{i}\right) \gamma(1)^{k} \beta\left(m_{1}, l_{1}\right) \prod_{i=2}^{p} \beta\left(m_{i}, l_{i}\right)+\Gamma \prod_{i=1}^{m} \alpha\left(n_{i}, j_{i}\right) \prod_{i=2}^{p} \beta\left(m_{i}, l_{i}\right) .
$$

Since $\Gamma=\sum_{i+j=1+k+2 m_{1}} a_{i} \gamma(1)^{j}$, where $a_{i} \in\left(N_{*}\right)_{i}$, the induction hypothesis and Case 3 finishes this case.

We have shown that monomials in our proposed generators along with those which split back from $N_{*}\left(H \times Z_{2} ; \mathcal{F}\right)$ will generate $N_{*}\left(H \times Z_{2}\right.$; All, $\mathscr{F})$ as an $N_{*}$ module. But $N_{*}(G ;$ All, $\mathscr{F}) \cong N_{*}(G) \oplus N_{*}(G ; \mathscr{F})$ via this splitting. This proves Theorem 2 . 


\section{REFERENCES}

1. J. C. Alexander, The bordism ring of manifolds with involutions, Proc. Amer. Math. Soc. 31 (1972), 536-542.

2. P. E. Connor and E. E. Floyd, Maps of odd period, Ann. of Math. (2) 84 (1966), 132-156.

3. R. J. Rowlett, Torsion in the bordism of oriented involutions, Trans. Amer. Math. Soc. 231 (1977), 541-548.

4. R. E. Stong, Unoriented bordism and actions of finite groups, Mem. Amer. Math. Soc. No. 103 (1970).

DepartMient of MATHEMatics, Indiana University, SOUTH BEND, INDIANA 46615 